\title{
PREDOMINO DE LA Brucella abortus EN CANES EN UN MUNICIPIO DE LA REGIÓN TROPICAL AMERICANA
}

\author{
Francisco Feliciano da SILVA JÚNIOR ${ }^{1}$; José Andreey Almeida TELES ${ }^{2}$; Rael Lucas \\ Fonseca de ALMEIDA; ${ }^{3}$; Gil Dutra FURTADO ${ }^{4}$; Aleudson dos Santos SILVA 5 ; Breno \\ Stefano Carvalho TABOSA ${ }^{6}$
}

\begin{abstract}
${ }^{1}$ Graduado en Medicina Veterinaria/Universidade Federal Rural de Pernambuco (UFRPE); Especialista en Clínica Médica de Rumiantes/Universidade Estadual Paulista Júlio de Mesquita Filho (UNESP); Maestro en Medicina Veterinaria Preventiva y Salud Pública/UNESP; Doctor en Medicina Veterinaria Preventiva y Salud Pública/UNESP. Médico Veterinario Autónomo, Brasil. E-mail: felicianojr@yahoo.com.br

${ }^{2}$ Graduado en Medicina Veterinaria/Universidade Federal de Campina Grande (UFCG); Especialista en Dolencias Infecto-contagiosas de los Animales Domésticos/UFRPE; Maestro en Biociencia Animal/UFRPE; Doctor en Ciencia Veterinaria/UFRPE. Coordinador y Docente del Curso de Medicina Veterinária da Faculdade Maurício de Nassau, Brasil. E-mail: teles.jaa@gmail.com

${ }^{3}$ Graduado en Medicina Veterinaria/Centro Universitario Cesmac; Pós-graduando en Clínica Médica y Cirúrgica de Pequeños Animales/Instituto Qualittas de Pós-Graduação, Brasil. E-mail: raellucas@gmail.com
\end{abstract}

${ }^{4}$ Graduando en Medicina Veterinaria/Faculdade Maurício de Nassau (UNINASSAU/PB); Ingeniero Agrónomo/Universidade Federal da Paraíba (UFPB); Doctor en Psicobiologia/Universidade Federal de Rio Grande do Norte (UFRN); Agrônomo-Sócio da Cooperativa de Agronegócio (COOPAGRO), Brasil. E-mail: gdfurtado@hotmail.com

${ }^{5}$ Graduando en Medicina Veterinaria de la Facultad Mauricio de Nassau, Brasil. E-mail: aleudson@gmail.com ${ }^{6}$ Graduado en Medicina Veterinaria/Centro Universitario Cesmac; Médico Veterinario Autónomo, Brasil

Resumen. La brucelosis canina es una enfermedad infecciosa de potencial zoonótico que afecta humanos, mamíferos domésticos y silvestres. La presencia de esta enfermedad en las hembras caninas, puede presentar muerte embrionaria precoz, abortamiento en el último tercio de gestación, altos índices de fetos expulsados muertos en el momento del parto; mientras que en los machos pueden presentar infertilidad, como consecuencia de alteraciones en los gametos masculinos, epididimitis, orquitis y dermatitis escrotal. También existen relatos de señales clínicas de inflamación intraocular, alteraciones en las vértebras, inflamación en las meninges, infección en los riñones y dermatitis pio granulomatosa. Generalmente es causada por la Brucella canis, y eventualmente, Brucella abortus, puede estar involucrada. Los métodos serológicos han sido los más utilizados para el diagnóstico de la brucelosis. El objetivo de este trabajo fue evaluar la seropredominancia de la $B$. abortus en canes del municipio de Sao Miguel dos Campos, Alagoas. La investigación utilizó 203 muestras de sangre, para obtención de suero. Estas fueron sometidas a las pruebas serológicas del Antígeno Acidificado Tamponado (AAT) en temperatura ambiente. De las 203 muestras, 31 fueron 
positivas al teste del AAT. La brucelosis, causada por B. abortus, es una enfermedad que está presente en canes del municipio de Sao Miguel dos Campos, Alagoas, siendo necesario que el poder público municipal tome providencias cuanto a la salud pública, teniendo en vista que se trata de una potencial zoonosis.

Palabras-clave: Brucelosis; Canes; Test Serológicos; Zoonosis.

\title{
PREVALENCE OF Brucella abortus IN DOGS OF THE COUNTY OF THE AMERICAN TROPICAL REGION
}

\begin{abstract}
Canine brucellosis is an infectious disease of zoonotic potential that affects humans, domestic and wild mammals. The presence of this disease in canine females may present early embryonic death, abortion in the final third of gestation, high rates of dead expelled fetuses on birth time, and in males may present infertility as a consequence of alterations in male gametes, epididymitis, orchitis and scrotal dermatitis. There are also reports of clinical signs of intraocular inflammation, changes in the vertebrae, inflammation in the meninges, infection of the kidneys and granulomatous pitting dermatitis. Usually it is caused by Brucella canis, and eventually, Brucella abortus, may be involved. Serological methods have been the most used for the diagnosis of brucellosis. The objective of this study was to evaluate the seroprevalence of B. abortus in dogs from the municipality of São Miguel dos Campos, AL. The study used 203 blood samples to obtain serum. These were submitted to the serological tests of Acidified Buffered Antigen (AAT) at room temperature. Of the 203 samples, 31 were positive to the AAT test. Brucellosis, caused by B. abortus, is a disease that is present in dogs of the municipality of São Miguel dos Campos, Alagoas, and it is necessary that the municipal public power should measure regarding public health, in view of being a potential zoonosis.
\end{abstract}

Keywords: Brucellosis; Dogs; Sorological tests; Zoonosis.

\section{PREVALÊNCIA DA Brucella abortus EM CÃES EM UM MUNICÍPIO DA REGIÃO TROPICAL AMERICANA}

Resumo. A brucelose canina é uma doença infectocontagiosa de potencial zoonótico que acomete os humanos, os mamíferos domésticos e silvestres. A presença desta doença nas fêmeas caninas, pode apresentar morte embrionária precoce, abortamento no terço final da gestação, altas taxas de fetos expelidos mortos no momento do parto, e nos machos podem apresentar infertilidade, como consequência de alterações nos gametas masculinos, epididimite, orquite e dermatite escrotal. Também existem relatos de sinais clinicos de inflamação intraocular, alterações nas vértebras, inflamação nas meninges, infecção nos rins e dermatite pio granulomatosa. Geralmente ela é causada pela Brucella canis, e eventualmente, Brucella abortus, pode estar envolvida. Os métodos sorológicos têm sido os mais utilizados para o diagnóstico da brucelose. O objetivo deste trabalho foi avaliar a 
soroprevalência da B. abortus em cães do município de São Miguel dos Campos, AL. A pesquisa utilizou 203 amostras de sangue, para obtenção do soro. Estes foram submetidos às provas sorológicas do Antígeno Acidificado Tamponado (AAT) em temperatura ambiente. Das 203 amostras, 31 foram positivas ao teste do AAT. A brucelose, causada por B. abortus, é uma enfermidade que está presente em cães do município de São Miguel dos Campos, Alagoas, sendo necessário que o poder público municipal tome providências quanto à saúde pública, tendo em vista tratar-se de uma zoonose em potencial.

Palavras-chave: Brucelose; Cães; Testes Sorológicos; Zoonoses.

\section{INTRODUCCIÓN}

Es cada vez más creciente el importante papel desempeñado por los canes en la sociedad, siendo frecuentemente asociados como guias para ciegos, guardianes y principalmente como acompañantes, tanto para niños y ancianos, como para los demás miembros de la familia (LARSSON, 1980; PORTO; CASSOL, 2007).

Son variadas las enfermedades que pueden afectar a los canes, siendo que algunas pueden causar problemas reproductivos al animal (hembra/macho), entre las cuales se resalta la brucelosis canina, zoonosis que trae grandes prejuicios al criador de canes (LUCERO, et al., 2010; MINHARRO, et al., 2005; SUTHERS-MCCABE, 2001).

La brucelosis canina es una enfermedad infecciosa de potencial zoonótico que afecta humanos, mamíferos domésticos y silvestres.

En los humanos, la enfermedad puede provocar muchos síntomas, variando de gripa leve hasta complicaciones graves en parte del sistema nervioso, sistema musculoesquelético y corazón, además de fiebre, dermatitis y linfadenopatía (GALIŃSKA; ZAGÓRSKI, 2013).

En los Estados Unidos son reportados anualmente de 100 a 200 casos de la infección en humanos (KAZMIERCZAK, 2012). En canes, provoca principalmente síntomas reproductivos y con menor frecuencia, linfadenopatía, hepato y esplenomegalia, meningoencefalitis, discoespondilitis y uveítis (HOLLETT, 2006; KAZMIERCZAK, 2012).

Generalmente la brucelosis canina es causada por la Brucella canis y, eventualmente, Brucella abortus, Brucella suis y Brucella melitensis, pueden estar involucradas (BRICKER, 2002). La principal fuente de infección por B. canis son los machos y las hembras enfermas mediante el contagio sexual o por la vía oral (ALMEIDA, et al., 2004; CFSPH, 2012; MOLNÁR; MOLNÁR; CARVALHO, 2001). 
Los canes también se presentan susceptibles a la infección por la B. abortus, principalmente en ambientes rurales, cuando entran en contacto con especies potencialmente afectadas como la bovina (CFSPH, 2012; RÍOS, 2003).

En varias situaciones, solamente es posible decir que una enfermedad infecciosa es diagnosticada con seguridad, despues del aislamiento e identificación del agente. Sin embargo, en el caso de muchas de esas enfermedades, eso es un proceso lento y muy oneroso, principalmente en medicina veterinaria, ya que es frecuente la necesidad de investigar muchos animales en programas de vigilancia (BADAKHSH; CARMICHAEL; DOUGLASS, 1982; JOHNSON; WALKER, 1992).

La brucelosis es tipicamente una enfermedad compleja, que exige tiempo para el aislamiento y la identificación del agente (7 a 14 días, además de ser un procedimiento de riesgo, por tratarse de una antropozoonosis (MOLNÁR; MOLNÁR; CARVALHO, 2001).

Debido a las limitaciones de los procedimientos laboratoriales que se apoyan en los cultivos, los métodos serológicos han sido los más utilizados para el diagnóstico de la brucelosis (MOLNÁR; MOLNÁR, CARVALHO, 2001).

Los métodos serológicos más utilizados para el diagnóstico como antígeno de pared celular son los test de seroaglutinación, principalmente la prueba lenta en tubo y la prueba rápida en placa (ALMEIDA, et al., 2001; CARMICHAEL; SHIN, 1996).

En esta perspectiva, el objetivo de este trabajo fue evaluar la seropredominancia de la Brucella abortus en canes del municipio de Sao Miguel dos Campos, Alagoas, Nordeste de Brasil, en la región tropical americana.

\section{MATERIAL Y MÉTODOS}

La investigación tuvo inicio en el municipio de Sao Miguel dos Campos, Estado de Alagoas, Nordeste del Brasil (Fig. 1), con la colección de 203 muestras de sangre, buscando levantamiento de la seropredominancia por Brucella abortus. Estas muestras fueron procesadas en el Laboratorio de Enfermedades Infectocontagiosas de los Animales Domésticos, en la Clínica Escuela de Medicina Veterinaria del Centro Universitario CESMAC. 


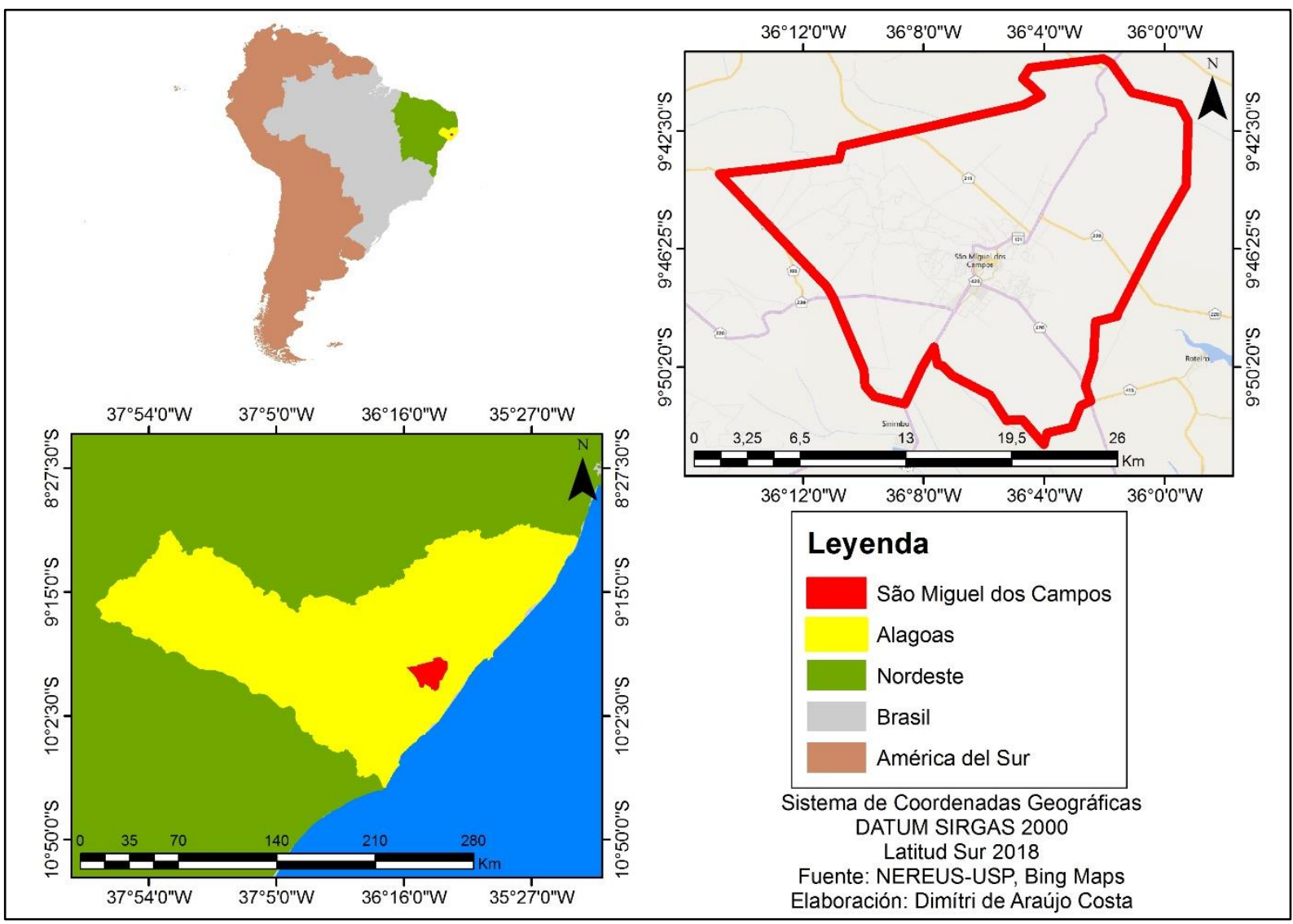

Figura 1. Mapa de localización de la ciudad de São Miguel dos Campos, Estado de Alagoas, Nordeste del Brasil.

Las muestras de sangre fueron recolectadas por punción venosa de la cefálica o yugular con agujas 25x7, precedidas de antisepsia con alcohol yodado en la región. El volumen de sangre obtenido $( \pm 3 \mathrm{~mL})$ fue recolectado en tubos de ensayo de $5 \mathrm{~mL}$ que permanecieron inclinados para facilitar el proceso de retracción del coágulo, buscando obtener suero para la realización de los test serológicos. Los sueros fueron transferidos para microtubos estériles de polipropileno, y mantenidos congelados a $-20^{\circ} \mathrm{C}$ hasta la realización de los test serológicos.

En el momento de la realización de las pruebas serológicas, las muestras fueron descongeladas y mantenidas a temperatura ambiente. Todas las muestras fueron testadas frente al Antígeno Acidificado Tamponado (AAT), según Brasil (2006) y serían sometidas a las Inmunodifusión en Gel de Agar (IDGA), siguiendo las instrucciones del fabricante (KEID, et al., 2007). 


\section{Antígenos para Evaluación Serológica}

La prueba del Antígeno Acidificado Tamponado (AAT) fue realizada con base en la metodología del Programa Nacional de Control y Erradicación de Brucelosis y Tuberculosis PNCEBT (BRASIL, 2006), utilizando antígeno constituido por suspensión celular inactivada de B. abortus (muestra 119-3), en la concentración de $8 \%, \mathrm{pH} 3,63$, colorado con rosa bengala, de la siguiente forma:

E suero y el antígeno permanecieron en temperatura ambiente por 30 minutos antes de realizarse la prueba. Fueron depositadas en placa cuadriculada padrón, $30 \mu \mathrm{L}$ de suero a ser testado y a su lado $30 \mu \mathrm{L}$ del antígeno. La homogenización del suero y el antígeno fue realizada con bastón de vidrio, formando círculos de aproximadamente $2 \mathrm{~cm}$ de diámetro. La placa fue agitada con movimientos oscilatorios continuos durante 4 minutos, en una frecuencia de aproximadamente 30 movimientos por minutos, de modo que permitiera a la mezcla suero-antígeno, fluir lentamente dentro de cada círculo (CASTRILLÓN-SALAZAR et al, 2013).

La lectura fue realizada al finalizar este periodo y los resultados interpretados, a partir de la reacción de aglutinación, indicados por la formación de grumos en los animales positivos y ausencia de los mismos en los animales negativos.

La muestra fue compuesta por 203 especímenes, considerando un límite de confianza de $95 \%$ y un error de estimativa de predominio del 5\% (ZAR, 1996).

\section{RESULTADOS Y DISCUSIÓN}

En este estudio fueron recolectadas, de forma aleatoria, 203 muestras de sangre de canes, siendo $126(62,06 \%)$ machos y $77(37,93 \%)$ hembras. Los animales tenían edades que variaban entre 1 mes hasta 8 años.

De las 203 muestras evaluadas, $31(15,27 \%)$ fueron positivas, frente al test del Antígeno Acidificado Tamponado (AAT), valor superior al de Azevedo et al. (2003), que obtuvo 6,77\% de reactivos, con un total de 118 animales en Paraíba. En otro trabajo con canes en el estado de Minas Gerais, también utilizando el AAT, se detectó un predominio de $18,1 \%$ para $B$. abortus, de un total de 635 canes, y también se obtuvo 14,2\% de predominio para B. canis (ALMEIDA et al., 2004). Cavalcanti et al. (2006) revelaron, en el estado de Bahía, 5,88\% de animales reactivos al IDGA, de 85 muestras analizadas. Aguiar et al. (2005), en el estado de Rondonia, obtuvieron predominio de 18,4\% de animales reactivos al AAT de 304 canes (DORNELES et al., 2011; GODOY; PERES, BARG, 1977). 
De los 31 animales positivos para B. abortus, 18 (58,06\%) animales eran machos y 13 (41,93\%) hembras. Almeida et al. (2004) no observaron diferencia significativa entre sexos, siendo $50 \%$ de machos y 48,6\% de hembras, y 1,4\% de los propietarios no declararon el sexo de los animales.

Así como Cavalcanti et al. (2006) en su investigación con B. canis, $40 \%$ de los animales reactivos eran machos y $60 \%$ hembras, tampoco evidenciando ninguna significación estadística referente a la predisposición del sexo de los individuos.

De las 13 hembras reactivas frente al AAT, 1 (7,69\%) tuvo histórico de aborto, acordando con la literatura que cita que la brucelossis canina causa abortos, principalmente en el tercio final de la gestación (SUZUKI et al., 2008).

En ese panorama, deben tomarse los debidos cuidados en la manipulación de los animales, bien como identificar y eliminar los animales con resultado positivo frente a los exámenes laboratoriales (SUZUKI et al., 2008).

\section{CONCLUSIONES}

La brucelosis canina es una enfermedad presente en canes del municipio de San Miguel dos Campos, Alagoas; siendo necesario que el poder público municipal tome providencias referentes a la salud pública, teniendo en vista que se trata de una potencial zoonosis. Tales resultados refuerzan la necesidad del Médico Veterinario prestar atención, cada vez más, a esta enfermedad, teniendo en vista su importancia económica y para la salud pública.

\section{AGRADECIMENTOS}

Los autores agradecen a la "Clínica Escola de Medicina Veterinária", do "Centro Universitário" CESMAC.

\section{REFERENCIAS}

AGUIAR, D.M.; CAVALCANTE, G.T.; VASCONCELLOS, S.A.; MEGID, J.; SALGADO, V.R.; CRUZ, T.F.; LABRUNA, M.B.; PINTER, A.; SILVA, J.C.R.; MORAES, Z.M.; CAMARGO, L.M.A.; GENNARI, S.M. Ocorrência de anticorpos anti-Brucella abortus e anti-Brucella canis em cães rurais e urbanos do Município de Monte Negro, Rondônia, Brasil. Revista Ciência Rural, Santa Maria, v. 35, n. 5, p. 1216-1219, 2005.

ALMEIDA, A.C.; SANTORELLI, A.; BRUZADELLI, R.M.Z.; OLIVEIRA, M.M.N.F. Soroepidemiologia da brucelose canina causada por Brucella canis e Brucella abortus na 
cidade de Alfenas, MG. Arquivo Brasileiro de Medicina Veterinária e Zootecnia, Belo Horizonte, v. 56, n. 2, p. 275-276, 2004.

ALMEIDA, A.C.; MENESES, A.M.; BERNIS, V.M.O.; SOARES, T.M.P.; LOIOLA, C.F.; MARINOVICK, C.; FERREIRA, P.A.S. Soroprevalência de brucelose canina na cidade de Alfenas, MG. Dados preliminares. Arquivo Brasileiro de Medicina Veterinária e Zootecnia, Belo Horizonte, v. 53, n. 3, p. 358-360, 2001.

AZEVEDO, S.S.; BATISTA, C.S.A.; ALVES, C.J.; CLEMENTINO, I.J. Ocorrência de anticorpos contra Brucella abortus em cães errantes da cidade de Patos, estado da Paraíba, Brasil. Arquivos do Instituto Biológico, São Paulo, v. 70, n. 4, p. 449-500, 2003.

BADAKHSH, F.F.; CARMICHAEL, L.E.; DOUGLASS, J.A. Improved rapid slide agglutination test for presumptive diagnosis of canine brucellosis. Journal of Clinical Microbiology, v. 15, n. 2, p. 286-289, 1982.

BRASIL. Programa nacional de controle e erradicação da brucelose e tuberculosePNCEBT. Ministério da Agricultura, Pecuária e Abastecimento. 2006. 125 p.

BRICKER, B.J. PCR as a diagnostic tool for brucellosis. Veterinary Microbiology, v. 90, p. 435-446. 2002.

CARMICHAEL, L.E.; SHIN, S.J. Canine brucellosis: a diagnostician's dilemma. Seminars in Veterinary Medicine Surgery (Small Animal), Orlando, v. 11, n. 3, p. 161-165, 1996.

CASTRILLÓN-SALAZAR， L.; GIRALDO-ECHEVERRI， C.A.; SÁNCHEZ-JIMÉNEZ, M.M.; OLIVEIRA-ANGEL, M. Factors associated with Brucella canis seropositivity in kennels of two regions of Antioquia, Colombia. Cadernos de Saúde Pública, v. 29, n. 10, p. 1975-1987, 2013.

CAVALCANTI, L.A.; DASSO, M.G.; OLIVEIRA, F.C.S.; VIEGAS, S.A.R.A.; ALMEIDA, M.G.A.R.; ANUNCIAÇÃO, A.V.M.; ALCANTARA, A.C.; BITTENCOURT, D.V.V.; OLIVEIRA, E.M.D. Pesquisa de anticorpos anti-Brucella canis em cães provenientes da 
região metropolitana de Salvador. Revista Brasileira de Saúde e Produção Animal, Salvador, v. 7, n. 2, p. 176-180, 2006.

CFSPH (The Center for Food Security \& Public Health). Brucellosis: Brucella canis Contagious Abortion, Undulant Fever. Institute for International Cooperation in Animal Biologics, 2018. Available on: http://www.cfsph.iastate.edu/Factsheets/pdfs/brucellosis_canis.pdf. Accessed on: 10 Oct. 2018.

DORNELES, E.M.S.; SANTOS, H.; MINHARRO, S.; NASCIMENTO-ROCHA, J.M.; MATHIAS, L.A.; DASSO, M.G.; TIENSOLI, C.D.; HEINNEMAN, M.B.; LAGE, A.P. Anticorpos anti-B. canis e anti-B. abortus em cães de Araguaína, Tocantins. Brazilian Journal Veterinary Research Animal Science, São Paulo, v. 48, n. 2, p. 167-171, 2011.

GALIŃSKA, E.M.; ZAGÓRSKI, J. Brucellosis in humans - etiology, diagnostics, clinical forms. Annals of Agricultural and Environmental Medicine. v. 20, n. 2, p. 233-238. 2013.

GODOY, A.M.; PERES, J.N.; BARG, L. Isolamento de Brucella canis em Minas Gerais, Brasil. Arquivos da Escola de Veterinária da Universidade Federal de Minas Gerais, Belo Horizonte, v. 29, p. 35-42, 1977.

HOLLETT, R.B. Canine brucellosis: outbreaks and compliance. Theriogenology, v. 66, n. 3, p. $575-587,2006$.

JOHNSON, C.A.; WALKER, R.D. Clinical signs and diagnosis of B. canis infection. The Compendium on Continuing Education Practician Veterinary, v. 14, n. 6, p. 763-772, 1992.

KAZMIERCZAK J. Public Health Implications of B. canis Infections in Humans. Summary Findings and Recommendations of the B. canis. DVM, MS; Wisconsin Division of Public Health; Chairperson. Workgroup: National Association of State Public Health Veterinarians, 2012. 
KEID, L.B.; SOARES, R.M.; VASCONCELLOS, S.A.; CHIEBAO, D.P.; MEGID, J.; SALGADO, V.R.; RICHTZENHAIN, L.J. A polymerase chain reaction for the detection of Brucella canis in semen of naturally infected dogs. Theriogenology., v. 67, n. 7, p. 1203 1210, 2007.

LARSSON, M.H.M.A. Pesquisa de aglutininas anti-Brucella canis em soros humanos na cidade de São Paulo, Brasil. Revista de Saúde Pública, São Paulo, n. 14, p. 404-407, 1980.

LUCERO, N.E.; CORAZZA, R.; ALMUZARA, M.N.; REYNES, E.; ESCOBAR, G.I.; BOERI, E.; AYALA, S.M. Human Brucella canis outbreak linked to infection in dogs. Epidemiology \& Infection, New York and Cambridge, v. 138, n. 2, p. 280-285, 2010.

MINHARRO, S.; et al. Diagnóstico da brucelose canina: dificuldades e estratégias. Revista Brasileira de Reprodução Animal. 29(3/4):167-173. 2005.

MOLNÁR, E.; MOLNÁR, L.; CARVALHO, M. Capacidade de algumas provas sorológicas no diagnóstico de brucelose canina. A Hora Veterinária, v. 21, n. 121, p. 45-49, 2001.

PORTO, R.T.C.; CASSOL, S. Zooterapia uma lição de cidadania: o cão sociabilizador e a criança vítima da violência intrafamiliar. Universidade de Santa Cruz do Sul - UNISC. Revista de Discussão Jurídica Campo Mourão, v. 3, n. 2, p. 46-74, 2007.

RÍOS, A.P. Mascotas en los hogares: enfermedades de los niños adquiridas por convivencia con animales. Enfermedades Infecciosas y Microbiología, Ciudad de México, v. 23, n. 4, p. 137-148, 2003.

SUZUKI, E.Y.; PENHA, G.A.; UEDA, F.S.; SALVARANI, R.S.; ALVES, M.L.; ZAPPA, V. Brucelose canina: revisão de literatura. Revista Científica Eletrônica de Medicina Veterinária, Garça, n. 10, ano 6, 2008.

ZAR, J.H. Bioestatistical Analysis. Prentice-Hall. New Jersey. 718p. 1996. 